\title{
Second Harmonic Generation, Beam Dynamics and Spatial Soliton Generation in Periodically Poled KTiOPO
}

\author{
H. Kim, L. Jankovic and G. Stegeman \\ School of Optics/CREOL, University of Central Florida, USA \\ S. Carrasco And L. TORner \\ Institute of Photonic Sciences, University Politecnica de Catalunya \\ Barcelona, Spain \\ M. Katz AND D. Eger \\ Electro-Optic Div., Soreq NRC, Israel

\begin{abstract}
Spatial solitons were investigated in periodically poled $\mathrm{KTiOPO}_{4}$ under conditions of second harmonic generation and many new features associated with soliton generation in quasi-phase-matched samples were observed. The effects on the second harmonic generation tuning curves of the beam narrowing mechanisms responsible for soliton generation were found to be dramatic. It was demonstrated experimentally that the mutual collapse of the fundamental and harmonic beams is a complex phenomenon.
\end{abstract}

PACS numbers: $42.65 . \mathrm{Jx}, 42.65 . \mathrm{Ky}, 42.65 . \mathrm{Tg}$

\section{Introduction}

The family of spatial solitons, i.e. beams of finite cross-section which propagate without diffraction has grown substantially in the last decade [1]. Quadratic spatial solitons are unique members of the soliton family because they require the co-existence of two (or even three) frequency components mutually coupled through the second order nonlinearity $d_{i j k}^{(2)}[1-3]$. They have been observed in 
both waveguides and bulk media and many of their properties are now well understood [4-10]. The threshold intensities for spatial soliton generation depend inversely on the effective nonlinearity used, $d_{\mathrm{eff}}^{(2)}$. It is well known in the nonlinear optics community that the most efficient frequency conversion is obtained with

periodically poled materials, with $\mathrm{LiNbO}_{3}(\mathrm{PPLN})$ for which $d_{\mathrm{eff}}^{(2)}>15 \mathrm{pm} / \mathrm{V}$ and indeed the lowest quadratic soliton thresholds have been reported in PPLN. However, $\mathrm{LiNbO}_{3}$ suffers from photorefractive damage and usually requires operation at temperatures $>100^{\circ} \mathrm{C}$, especially when high intensities are used for soliton studies where temperatures in excess of $150^{\circ} \mathrm{C}$ are needed [5].

Another ferroelectric, namely $\mathrm{KTiOPO}_{4}$, does not suffer from photorefractive damage, even at room temperature. It has been periodically poled (to produce periodically poled $\mathrm{KTiOPO}_{4}$ (PPKTP)) and second harmonic generation (SHG) has been reported [11-13]. The optical quality of such crystals is excellent and SHG experiments have yielded large conversion efficiencies. This should lead to reasonably low soliton thresholds of a few $\mathrm{GW} / \mathrm{cm}^{2}$. Here we review our experiments on the properties of quadratic solitons in PPKTP and their impact on SHG efficiency and tuning curves.

\section{Samples and experimental apparatus}

KTP is a biaxial ferroelectric crystal. Its ferroelectric domains, and hence the second order nonlinearity were periodically reversed along the $z$-axis at Soreq using the low temperature poling technique [11]. When $z$-polarized light is propagated along the crystal's $x$-axis, phase-matching can be achieved when the periodicity $\Lambda$ satisfies the wave vector matching condition $\Delta k=0$ with $\Delta k=$ $2 k(\omega)-k(2 \omega)+2 m \pi / \Lambda$, where $m$ is an integer, and $k(2 \omega)$ and $k(\omega)$ are the wave vectors at the harmonic and fundamental frequencies respectively. The effective nonlinear coefficient has been measured to be $9.5 \mathrm{pm} / \mathrm{V}\left(d_{\mathrm{eff}}^{(2)}=2 d_{33}^{(2)} / \pi\right)$ which corresponds to a $d_{33}^{(2)}=14.9 \mathrm{pm} / \mathrm{V}[12,13]$. The sample was $1 \mathrm{~cm}$ long and the width and thickness of the PPKTP were $4.5 \mathrm{~mm}$ and $0.5 \mathrm{~mm}$ (along $z$ ) respectively. The measured SHG band width was $0.2 \mathrm{~nm}$, in good agreement with theory and an indication that phase-matching is realized over the full length of the crystal $[12,13]$.

Because both the propagation and polarizations lie along the crystal axes of a biaxial crystal, the phase-matching is non-critically-phase-matched (NCPM). This is indicated in Fig. 1 where the effect of the QPM is to move the appropriate cut of the fundamental refractive index surface to tangency with the second harmonic one, case (b). For a poling period of $8.99 \mu \mathrm{m}$ and $m=1$, phase-matching for a $1064 \mathrm{~nm}$ fundamental beam occurs around $42.7^{\circ} \mathrm{C}$ [14]. At temperatures lower than the phase-matching temperature $\left(T<T_{\mathrm{PM}}\right.$, Fig. 1a), there are two directions symmetrically displaced in angle from the $x$-axis at which phase-matching occurs, whereas for $T>T_{\mathrm{PM}}$ the interaction cannot be phase-matched at any angle. 
Let us note that for on-axis propagation, there is no spatial walk-off between the fundamental and harmonic beams. However, for propagation at an angle $\theta$ from the $x$-axis, the walk-off is dominated by the QPM condition giving an angular walk-off $\psi=\sin ^{-1}[\sin \theta 2 \pi / k(2 \omega) \Lambda][15]$.

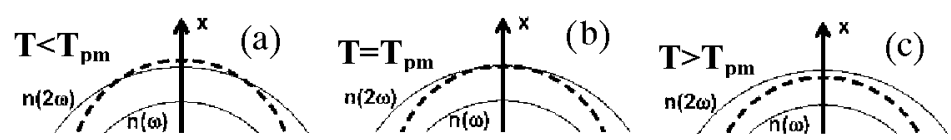

Fig. 1. Cuts of the index ellipsoids in the $x-y$ plane for the $z$-polarized FW and SH. The dashed line is meant to simulate approximately the effect of the nonlinear QPM grating on phase-matching. Part (a) is for temperatures below $T_{\mathrm{pm}}$, (b) is at the phase-matching temperature, (c) $T>T_{\mathrm{pm}}$.

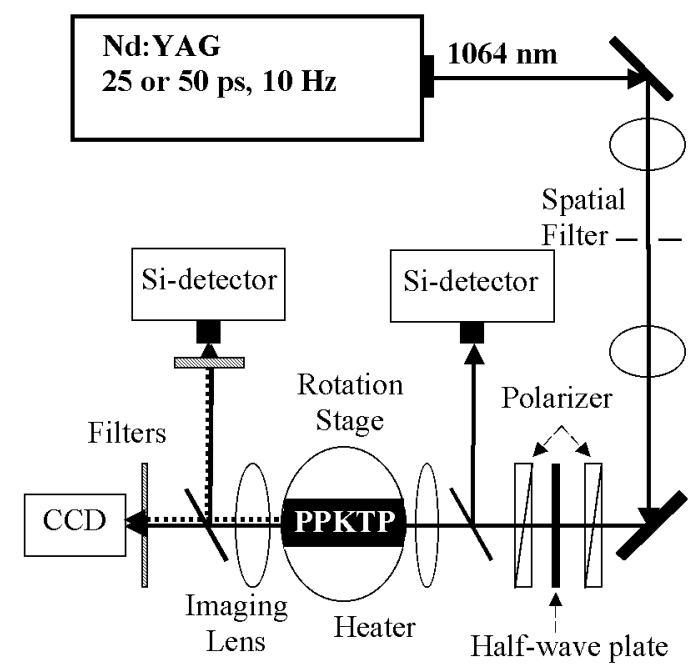

Fig. 2. Measurement apparatus and the parameters for the laser system and beams.

The experimental system used is shown schematically in Fig. 2. The laser source was an EKSMA Nd:YAG $10 \mathrm{~Hz}$ system. Pulse energies of only a few $\mu \mathrm{J}$ were required for the experiments. The output beam from the laser was spatially filtered giving a measured Gaussian beam quality factor $M^{2}$ of $1.0 \approx 1.1$. The laser's full-width half maximum spectral band width was measured to vary from 0.11 (50 ps pulses) to $0.14 \mathrm{~nm}$ ( 25 ps pulses), about a factor of two smaller than the SHG band width.

The fundamental beam was focused onto the input crystal facet by lenses to minimum beam waists $w_{0}=40$ (for approximating the plane wave limit) and $\approx 16-18 \mu \mathrm{m}$ (for the narrow beam experiments), as measured by the knife-edge 
method. A minimum waist of $16.4 \mu \mathrm{m}$ gives 6.9 diffraction lengths of propagation at $M^{2}=1$. The individual beams emerging from the back face of the crystal were directed onto a camera and a detector to measure their pulse energy and spatial distribution.

\section{Physics of SHG beam dynamics}

In order to understand the SHG and soliton generation at high intensities, it is necessary to first understand how the combination of beam nonlinearity and propagation affects the beam shape. As the input intensity is increased, two different nonlinear beam narrowing (or broadening) "mechanisms" come into play. The first is the universal narrowing mechanisms associated with all nonlinear wave mixing interactions [16]. In brief summary, in nonlinear optics the nonlinear polarization induced is always due to the product of fields. When the fields have finite cross-section, the nonlinear polarization derived from products of the fields is by definition narrower than the mixing fields and hence the electromagnetic waves generated are also narrower. For SHG by Gaussian beams for example, the generated harmonic (up-conversion) is $\sqrt{2}$ narrower than the fundamental. For down-conversion, which also always occurs, the regenerated fundamental is also narrower than either the fundamental or harmonic. Therefore this mechanism leads to both beams narrowed in space. If this mutual focusing is more effective than the usual diffraction, both beam cross-sections are reduced on propagation.

The second "mechanism" is called "cascading" [16, 17]. It is based on the difference between the fundamental and harmonic phase velocities which, in the case of ideal plane waves, occurs away from phase-match. On this regard, let us note that finite beams contain a continuous spectrum of plane waves, thus with finite pump beams cascading occurs also at nominal phase-matching. As energy flows back and forth between the two waves, the phase front of the slower wave retards that of the faster wave and effectively slows down the faster wave, i.e. it induces an additional phase shift. Since the amount of energy exchange depends on the local input intensity, the phase shift varies along the beam's transverse distribution, i.e. intensity profile. The resulting phase front becomes either concave or convex, leading to either a self-defocusing or self-focusing effect, respectively. Although in actual fact there is no refractive index change associated with this nonlocal effect, it can be interpreted as an effective nonlinear refractive index $n_{2}$, i.e. $n_{2, \text { eff }}$ with an equivalent index change $\Delta n_{\mathrm{eff}}=n_{2, \mathrm{eff}} I(r)$, where $I(r)$ is the radial dependence of the beam intensity. For $\Delta k>0$, self-focusing occurs and with $\Delta k<0$ defocusing takes place. Thus for $\Delta k>0$ beam narrowing is enhanced, whereas for $\Delta k<0$ the two "mechanisms" tend to interfere with one another. Let us notice that the simple picture sketched above obviously holds only in the limit where a large fundamental frequency signal interacts very weakly with its small second-harmonic, under conditions where the energy conversion between 
them is negligible. Otherwise, the above self-focusing self-defocusing picture does not hold. For example, under conditions of a second-harmonic comparable to the fundamental, self-focusing takes place at both sides of phase-matching, as was observed experimentally in the first observation of quadratic solitons [4].

It is now well understood that spatial solitons consisting of coupled fundamental and harmonic beams exist $[1-3,16,17]$. They are the nonlinear eigenmodes in the limit in which the diffraction length $L_{\mathrm{d}}=\pi w_{0}^{2} n / \lambda_{\text {vac }}$ is comparable to or larger than the parametric gain length $L_{\mathrm{pg}}=c n /\left[\omega d_{\mathrm{eff}}^{2}|E(\omega, x=0)|\right]$. Stable solitons for type I phase-match consist of in phase fundamental and harmonic components, with a specific harmonic to fundamental field ratio which depends on the input intensity and the initial wave vector mismatch $[1-3,16-19]$. Because they are the nonlinear eigenmodes, above a threshold intensity any input will evolve with distance into a quadratic soliton $[20,21]$. Typically only a fundamental input is used and the second-harmonic is allowed to evolve with distance under the influence of the usual coupled mode equations which describe SHG [4]. These equations lead near the input facet of the crystal to an $\mathrm{SH}$ beam which is $\pi / 2$ out of phase with the fundamental. Thus both the relative phase as well as the relative amplitudes of the FW and SH need to evolve into values appropriate to a quadratic soliton. This means that the well-known plane solutions for the $\mathrm{FW}$ and $\mathrm{SH}$, for example SHG $\propto \tanh ^{2}\left(z / L_{\mathrm{pg}}\right)$ on phase-match, are no longer valid. Instead the field amplitudes undergo oscillatory behavior which damps out with distance as the soliton properties are approached asymptotically. A typical example is shown in Fig. 3. Let us note that the exchange of energy occurs with distance between the FW and $\mathrm{SH}$ on phase-match. This process is not inherently adiabatic and the excess $\mathrm{FW}$ and $\mathrm{SH}$ energy is radiated away making the soliton generation efficiency less than $100 \%$

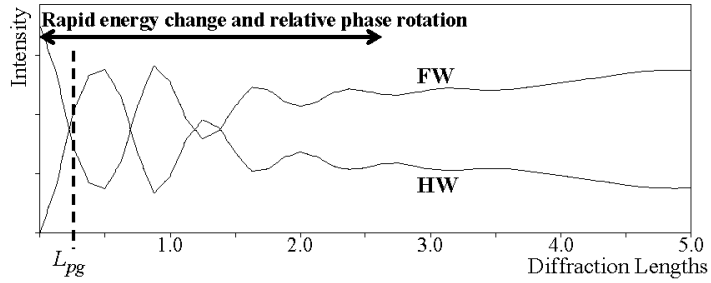

Fig. 3. Typical evolution of the intensity of a FW and a $\mathrm{SH}$ beam with propagation distance when only the FW is input.

The beam waist also undergoes more complex dynamics than monotonic narrowing of both beams [22]. Shown in Fig. 4 are the measured fundamental and harmonic beam waists at the output facet of the sample for various phase-mismatches [23]. The overall beam narrowing is clear. However, depending on the sign of the wave vector mismatch, the beam waist of one of the two beams initially becomes larger before it narrows down $[22,23]$. Let us note that the freely diffracting fun- 
damental is expected to have a width of $\approx 90 \mu \mathrm{m}$ at the end of the crystal. The incident intensity at which both beams, FW and $\mathrm{SH}$, reach a plateau with widths comparable to the input beam width is defined as the spatial soliton threshold [24]. For PPKTP on phase-match it occurs at a peak intensity of $\approx 3 \mathrm{GW} / \mathrm{cm}^{2}$ for an input $1 / \mathrm{e}^{2}$ beam size of $16.4 \mu \mathrm{m}$. However, let us note that the threshold for the nonlinear beam dynamics to set in is an order of magnitude smaller, of order $0.4 \mathrm{GW} / \mathrm{cm}^{2}$, or less.
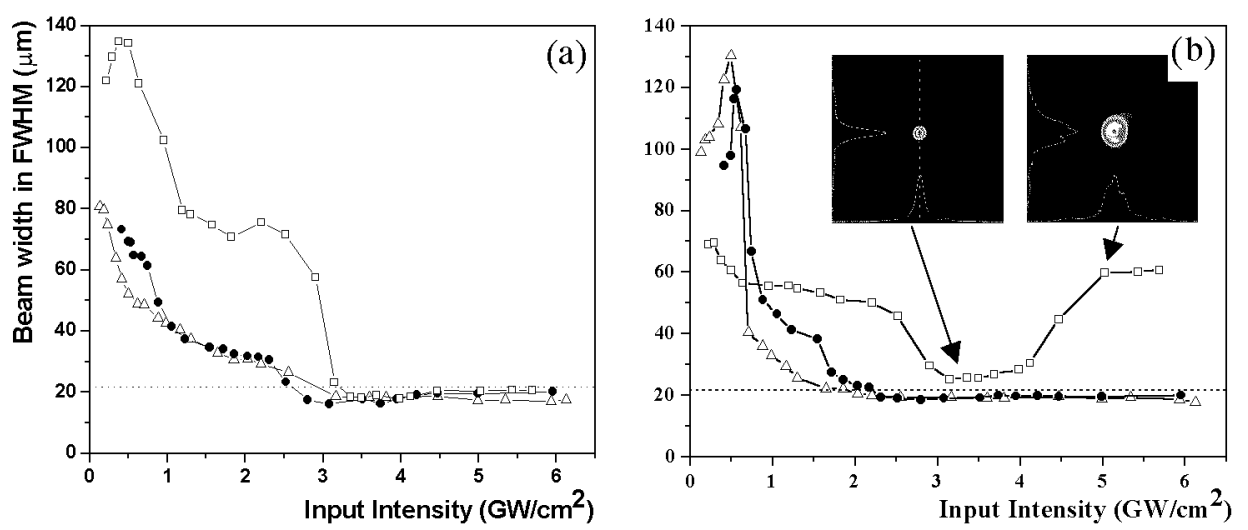

Fig. 4. FW and SH output beam sizes (FWHM) as a function of input intensity. (a) Fundamental, (b) second harmonic. In both (a) and (b), squares: $T=49.0^{\circ} \mathrm{C}$ $(\Delta k L=-2.4 \pi)$, circles: $T=42.7^{\circ} \mathrm{C}(\Delta k L=0)$, triangles: $T=37.5^{\circ} \mathrm{C}(\Delta k L=2.0 \pi)$. Conditions: $w_{0}=18.4 \mu \mathrm{m}$, pulse width $50 \mathrm{ps}, M^{2}=1.0$.

Away from phase-match, the details depend on whether the narrowing mechanisms interfere constructively or destructively, i.e. $\Delta k>0$ or $\Delta k<0$, respectively. Let us note that for $\Delta k>0$ solitons are formed at about the same threshold as for on phase-match, whereas for the equivalent $|\Delta k L|$ with $\Delta k<0$ solitons are not formed over the intensity range investigated. This is verified by both the intensity dependence of the harmonic output, and the output beam profiles. This behavior for negative phase-mismatch has been attributed to third order effects, Kerr nonlinear refraction and two photon absorption in KTP [23].

\section{SHG tuning curves and soliton generation}

The simplest experiment, usually the first one done with any nonlinear crystal, is to measure the SHG tuning curve at low input powers. Because the wavelength of the laser is fixed in the present case, the two options are to either vary the incidence angle at the phase-matching temperature, or to vary the temperature for incidence along the $x$-axis. These two tuning curves will give different results because the first exhibits symmetry about the $x$-axis, whereas the second has no symmetry about the phase-matching temperature, see Fig. 1. The unique 
aspect of the present experiments is that the input intensities used here are sufficiently large, and the input beam diameters small enough, to get significant beam narrowing over the length of the crystal and the complex beam dynamics that accompanies it.

\subsection{SHG temperature tuning curves}

When the input beam diameter is large enough so that the crystal length $L$ is at least one diffraction length long, the low power tuning curves resemble the usual $\operatorname{sinc}^{2}(\Delta k L / 2)$ response for both cases. The temperature tuning result is shown in Fig. 5a. However, when the input beam diameter is reduced, the difference in SHG response due to the different phase-mismatch conditions shown in Figs. 1a and $\mathrm{c}$ is evident. For $T<T_{\mathrm{pm}}$, there are two, symmetrically displaced with angle, phase-matching conditions. The angular spectrum associated with a narrow input beam has components which can therefore be phase-matched so that the SHG tuning curve has a broader response towards lower temperatures, again as exhibited in Fig. 5a. This asymmetry effect has been known since the early days of nonlinear optics $[25,26]$.
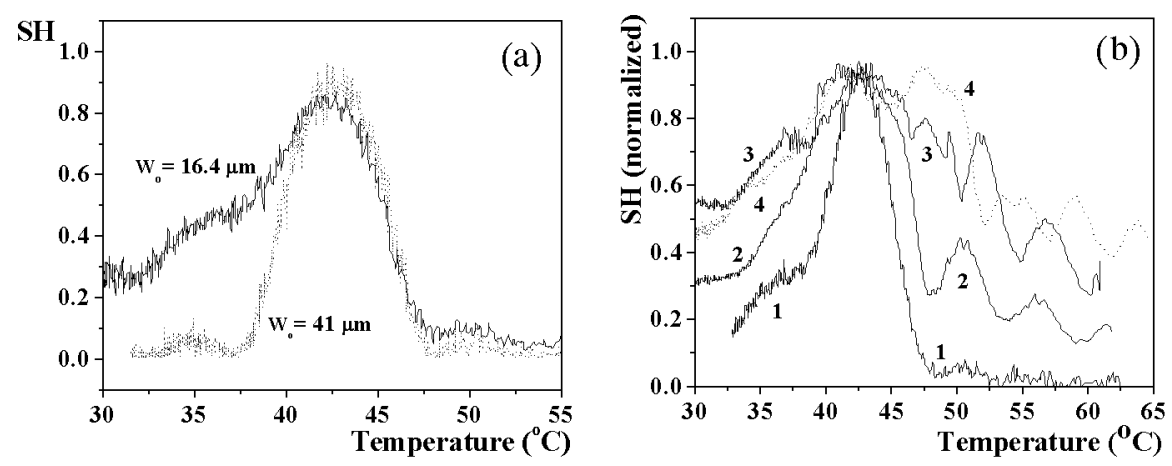

Fig. 5. Tuning curves for SHG obtained by temperature tuning around the low intensity phase-match temperature $\left(42.7^{\circ} \mathrm{C}\right)$. (a) Input intensity of $11 \mathrm{~kW} / \mathrm{cm}^{2}$ for two different input beam widths (solid line: $16.4 \mu \mathrm{m}$, dotted line: $41 \mu \mathrm{m}$ ). (b) Different input intensity: $1-0.02 \mathrm{GW} / \mathrm{cm}^{2}, 2-2.0 \mathrm{GW} / \mathrm{cm}^{2}, 3-6.1 \mathrm{GW} / \mathrm{cm}^{2}, 4-12.0 \mathrm{GW} / \mathrm{cm}^{2}$.

The SHG tuning curve on phase-match as a function of increasing input intensity is shown in Fig. 5b. The most salient feature is the broadening of the response curves with increasing intensity. The reason is caused by the self-phase modulation induced by cascading below the threshold for soliton formation and by the generation of spatial solitons above the threshold. Once a soliton is formed, it propagates unchanged to the end of the sample. Hence the SHG occurs effectively not over the whole sample, but over the distance required to establish a soliton. This requires a few parametric gain lengths $L_{\mathrm{pg}}$. Thus the effective length, at least 
as far as SHG is concerned, decreases with increasing intensity and becomes less than $L$. Hence the band width becomes larger than in the small conversion limit. This has been verified by detailed modeling [14].

When $\Delta k<0$, the situation is considerably more complex. As discussed before, $L_{\text {pg }}$ continues to decrease with increasing intensity. This leads to a progressively more rapid exchange of energy between the fundamental and harmonic with distance. Below the threshold for soliton formation, the two mechanisms, nonlinear optical beam narrowing via wave mixing and beam spreading due to cascading, compete with one another. Furthermore, the interference is different over different parts of the beam profile which leads to the large amount of "noise" on this side of the tuning curve relative to $\Delta k>0$ region. This results in very complex beam profiles. Let us note that at the highest input intensity recorded, this chaotic region actually leads to a maximum in the SHG which equals the on-phase match value for which soliton generation occurred.

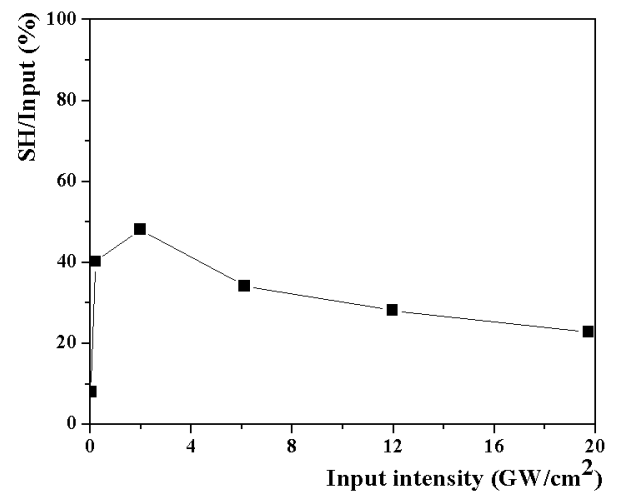

Fig. 6. SHG pulse energy efficiency versus input fundamental intensity at the QPM temperature $\approx 42.7^{\circ} \mathrm{C}$.

The variation in the SHG efficiency on phase-match is shown in Fig. 6. (The tuning curves discussed previously were normalized to the same maximum value.) The maximum SHG efficiency peaks at about $50 \%$, at about the soliton threshold. Let us note however that the SHG efficiency drops after it achieves its peak value. This is an indication that the conversion efficiency into solitons decreases once the soliton threshold is crossed and the excess fundamental electromagnetic energy is radiated away and not converted into spatially localized second harmonic.

\subsection{Angle tuning curves}

As mentioned previously, the $x$-axis being a crystal axis is also a symmetry axis. Thus for angle detuning away from the $x$-axis in one of the principal planes $x-y$ or $x-z$, the SHG properties, including quadratic solitons should be symmetrical about the $x$-axis. Results are shown for $\Delta k=0$ (Fig. 7), $\Delta k<0$ (Fig. 8) and 
$\Delta k>0$ (Fig. 9). The response curves all exhibit symmetry about the $x$-axis, as expected. They also all exhibit increases in angular band width for SHG with increasing input intensity which occurs for the same reasons as discussed previously for the temperature tuning case. Figure 7 indicates that at large input intensities on phase-match the band width opens up to about $\pm 8^{\circ}$ (external angle of incidence). Let us note, however, that as indicated in Fig. 6, the SHG efficiency drops with increasing intensity so that there is an efficiency-band-width trade-off.

Similar broadening etc. occurs for $\Delta k<0$, see Fig. 8 . The most interesting aspect of these curves is the minimum in the SHG that occurs along the $x$-axis

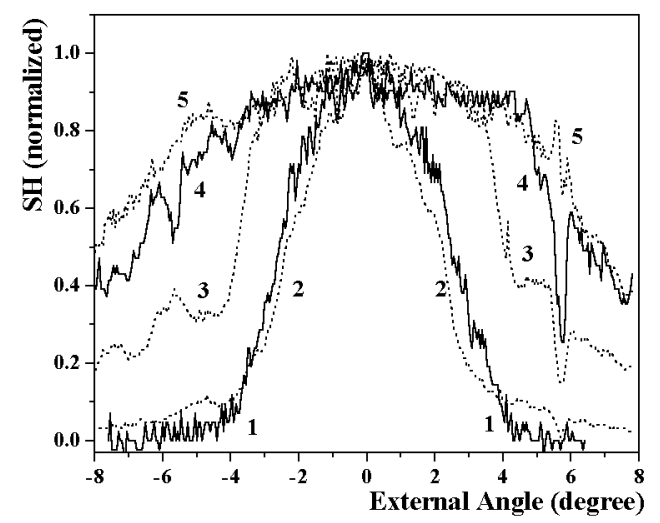

Fig. 7. SHG tuning curve versus incidence angle in the $x-y$ plane at $42.7^{\circ} \mathrm{C}$ $\left(T=T_{\mathrm{pm}}\right) \cdot 0^{\circ}$ angle corresponds to phase-match. Input intensity: $1-0.02 \mathrm{GW} / \mathrm{cm}^{2}$; $2-0.2 \mathrm{GW} / \mathrm{cm}^{2}, 3-2.0 \mathrm{GW} / \mathrm{cm}^{2}, 4-6.1 \mathrm{GW} / \mathrm{cm}^{2}, 5-19.7 \mathrm{GW} / \mathrm{cm}^{2}$.

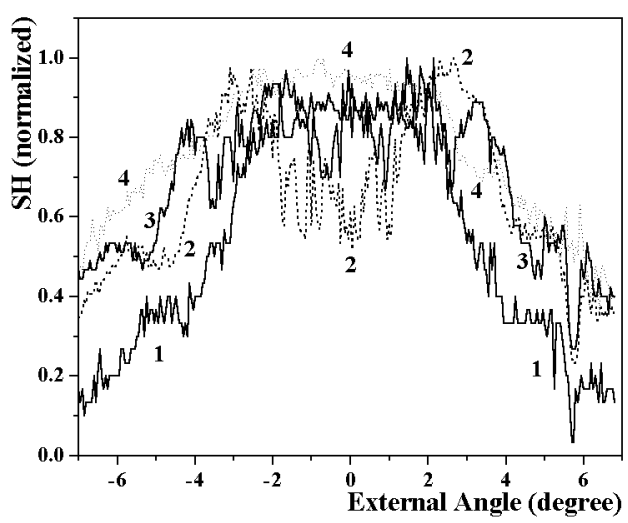

Fig. 8. SHG tuning curve versus incidence angle in the $x-y$ plane at $47.7^{\circ} \mathrm{C}\left(T>T_{\mathrm{pm}}\right)$. $0^{\circ}$ angle corresponds to the $x$-axis. Input intensity: $1-0.2 \mathrm{GW} / \mathrm{cm}^{2} ; 2-2.0 \mathrm{GW} / \mathrm{cm}^{2}$, $3-6.1 \mathrm{GW} / \mathrm{cm}^{2}, 4-19.7 \mathrm{GW} / \mathrm{cm}^{2}$. 
at a relatively low input intensity of $2.0 \mathrm{GW} / \mathrm{cm}^{2}$. At this intensity strong beam distortion has already set in and there is again an interplay between the cascading and wave mixing mechanisms so that energy oscillates between the fundamental and harmonic over distances comparable to the sample length. This interplay leads to the minimum along the poling axis at that particular input intensity. Let us note that there is no spatial soliton generation occurring at this intensity.

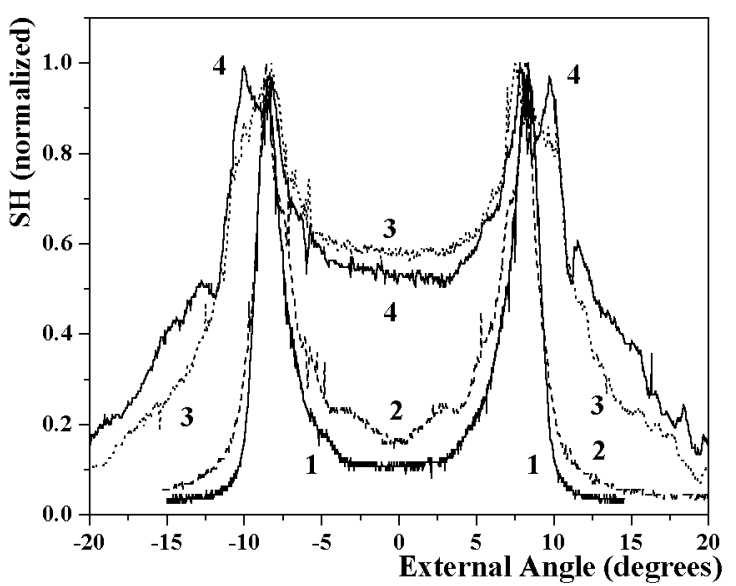

Fig. 9. SHG detuning curves at room temperature for different fundamental input intensities. $1-0.02 \mathrm{GW} / \mathrm{cm}^{2}, 2-0.9 \mathrm{GW} / \mathrm{cm}^{2}, 3-6.45 \mathrm{GW} / \mathrm{cm}^{2}, 4-12.9 \mathrm{GW} / \mathrm{cm}^{2}$.

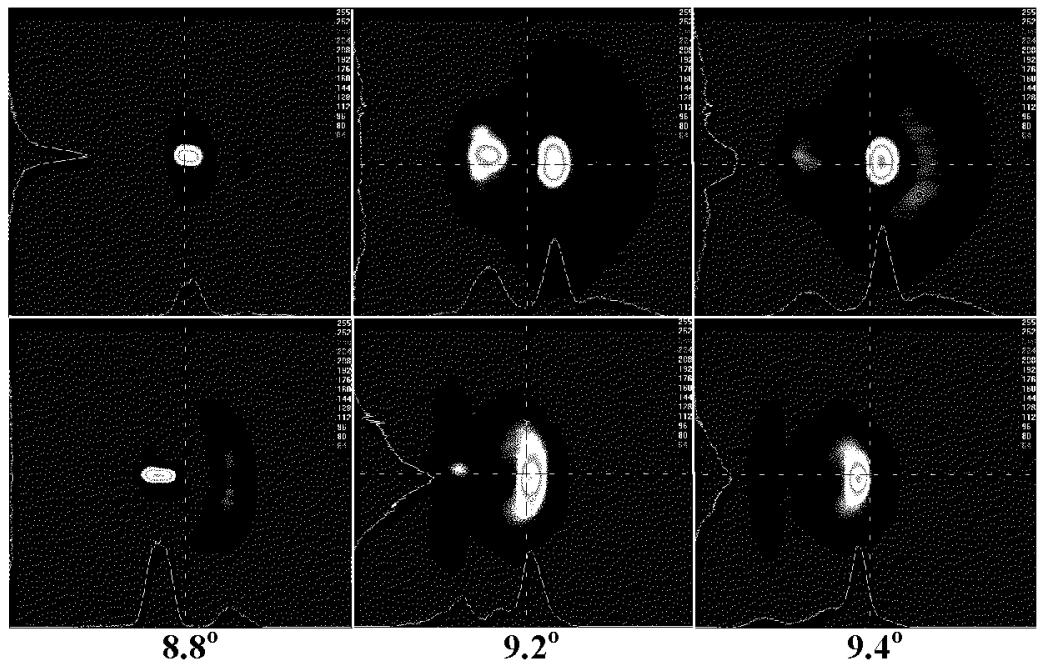

Fig. 10. Various output beam profiles, upper $\mathrm{FW}$ and lower $\mathrm{SH}$, on the original phase-matching peak $\left(8.8^{\circ}\right)$, the secondary peak $\left(9.4^{\circ}\right)$ and in the minimum between the two peaks $\left(9.2^{\circ}\right)$ for angle tuning at room temperature. The fundamental input intensity is $9.7 \mathrm{GW} / \mathrm{cm}^{2}$. 


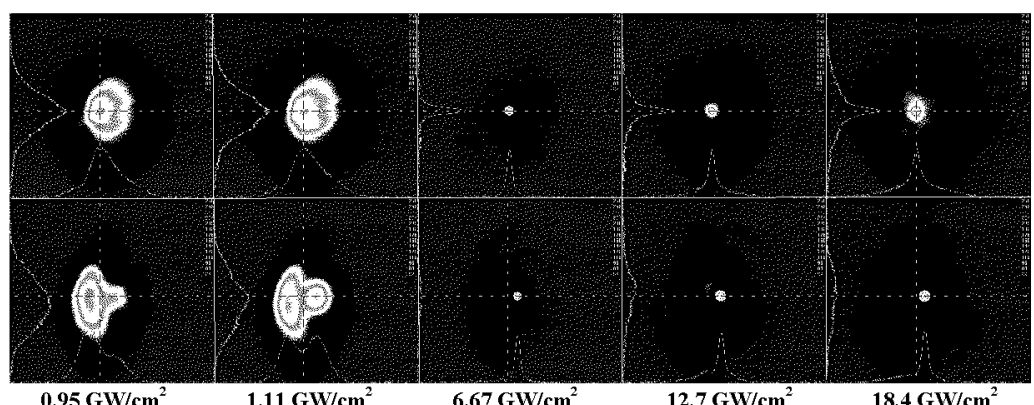

Fig. 11. A sampling of output beam profiles, upper FW and lower SH, for different input intensities on the phase-matching peak $\left(8.84^{\circ}\right)$ at room temperature.

Angular detuning corresponding to the scenario in Fig. 1a is shown in Fig. 9, i.e. $\Delta k>0$ along the $x$-axis. As expected, there are two maxima at $\pm 8.8^{\circ}$ corresponding to the two phase-matching conditions in Fig. 1a. Increasing input intensity again leads to broadening of the SHG response curves, a universal feature for all these results. At the highest intensities, the angular (external) band width for SHG is in excess of \pm 10 degrees. One of the more interesting features in these curves is the splitting of the peaks into doublets which occurs around $9.7 \mathrm{GW} / \mathrm{cm}^{2}$ input intensity. The output beams profiles obtained near the two peaks, and in the minimum between them, shown in Fig. 10, give some clues as to what is happening. As indicated previously, the QPM-induced walk-off occurs away from the $x$-axis, and the internal walk-off angle is about 0.15 degrees at these peaks. In fact it is clear from Fig. 10 that in the minimum region the fundamental which carries most of power is split spatially by the walk-off into two halves, and at the peaks it is a single beam.

The effects of the walk-off, the energy exchange between the fundamental and harmonic and finally soliton generation are clear in Fig. 11. At low intensities there is energy exchange between the beams and the walk-off leads to two FW and SH beams. The beam narrowing mechanisms tend to pull the different beams into a common spatial propagation direction [27]. This ultimately results in the generation of well-localized spatial solitons with a threshold of about $4 \mathrm{GW} / \mathrm{cm}^{2}$ with the occasional exception where the peaks are split as discussed previously.

\section{Summary}

The combination of high intensities of multi-GW/ $\mathrm{cm}^{2}$ and beams with waists in the 10's of microns leads to large changes in the output beam intensity distributions for harmonic generation in PPKTP. The threshold for soliton formation which occurs due to a balance between beam narrowing and normal diffraction is about $4 \mathrm{GW} / \mathrm{cm}^{2}$ at NCPM for 16.4 micron beam waists, Gaussian beam quality factor $M_{x}^{2} \approx M_{y}^{2} \approx 1.1$, at the input. Prior to and during soliton formation, 
there are a number of contributing mechanisms including diffraction, beam narrowing due to nonlinear wave mixing and cascading, wave vector mismatch and QPM-induced walk-off. The result is complex beam dynamics for both the fundamental and second harmonic.

The SHG efficiency peaks at about $50 \%$ on phase-match due to soliton generation and then falls with increasing intensity as the efficiency of soliton generation decreases slowly. The band widths of the SHG tuning curves always increase with increasing intensity since the parametric gain length which varies inversely with the input field limits the length over which the SH signals grow.

\section{Acknowledgments}

The experimental research in the US was supported by an Army Research Office MURI, and the collaboration between the American and Spanish investigators was made possible by the Commission for Scientific Exchange between the USA and Spain. S.C. and L.T. were supported by the Generalitat de Catalunya and by the Spanish Government under grant TIC2000-1010.

\section{References}

[1] Reviewed recently in Spatial Solitons, Ed. S. Trillo, W. Torruellas, Springer-Verlag, Berlin 2001.

[2] Yu.N. Karamzin, A.P. Sukhorukov, Zh. Eksp. Teor. Phys. 68, 834 (1975) (Sov. Phys.-JETP 41, 414 (1976)).

[3] G.I. Stegeman, in: Proc. NATO Advanced Research Workshop on Soliton Driven Photonics, Ed. A.D. Boardman, A.P. Sukhorukov, Kluwer Academic Publishers, Holland 2001, p. 21.

[4] W.E. Torruellas, Z. Wang, D.J. Hagan, E.W. VanStryland, G.I. Stegeman, L. Torner, C.R. Menyuk, Phys. Rev. Lett. 74, 5036 (1995).

[5] R. Schiek, Y. Baek, G.I. Stegeman, Phys. Rev. E 53, 1138 (1996).

[6] P. Di Trapani, G. Valiulis, W. Chianglia, A. Adreoni, Phys. Rev. Lett. 80, 265 (1998).

[7] M.T.G. Canva, R.A. Fuerst, D. Baboiu, G.I. Stegeman, G. Assanto, Opt. Lett. 22, 1683 (1997).

[8] B. Bourliaguet, V. Couderc, A. Barthelemy, G.W. Ross, P.G.R. Smith, D.C. Hanna, C. De Angelis, Opt. Lett. 24, 1410 (1999).

[9] X. Liu, L.J. Qian, F.W. Wise, Phys. Rev. Lett. 82, 4631 (1999).

[10] R. Malendevich, L. Jankovic, S. Polyakov, R. Fuerst, G.I. Stegeman, Ch. Bosshard, P. Gunter, Opt. Lett. 27, 631 (2002).

[11] G. Rosenman, A. Skliar, D. Eger, M. Oron, M. Katz, Appl. Phys, Lett. 73, 3650 (1998).

[12] A. Englander, R. Lavi, M. Katz, M. Oron, D. Eger, E. Lebiush, G. Rosenman, A. Skliar, Opt. Lett. 22, 1598 (1997). 
[13] A. Arie, G. Rosenman, A. Korenfield, A. Skliar, M. Oron, M. Katz, D. Eger, Opt. Lett. 23, 28 (1998).

[14] H. Kim, L. Jankovic, G.I. Stegeman, M. Katz, S. Carrasco, L. Torner, Appl. Phys. Lett. 81, 2710 (2002).

[15] M.M. Fejer, G.A. Magel, D.H. Jundt, R.L. Byer, IEEE J. Quant. Electron. 28, 2631 (1992).

[16] Reviewed in G.I. Stegeman, D.J. Hagan, L. Torner, Opt. Quantum Electron. 28, 1691 (1996).

[17] G. Assanto, G.I. Stegeman, Opt. Express 10, 388 (2002).

[18] L. Torner, Opt. Commun. 114, 136 (1995).

[19] L. Torner, D. Mihalache, D. Mazilu, E.M. Wright, W.E. Torruellas, G.I. Stegeman, Opt. Commun. 121, 149 (1995).

[20] L. Torner, C.R. Menyuk, G.I. Stegeman, Opt. Lett. 19, 1615 (1994).

[21] L. Torner, E.M. Wright, J. Opt. Soc. Am. B 13, 864 (1996).

[22] P. Di Trapani, A. Bramati, S. Minardi, W. Chinaglia, C. Conti, S. Trillo, J. Kilius, G. Valiulis, Phys. Rev. Lett. 87, 183902 (2001).

[23] H. Kim, L. Jankovic, G.I. Stegeman, S. Carrasco, L. Torner, D. Eger, M. Katz, Opt. Lett., in press.

[24] R.A. Fuerst, B.L. Lawrence, W.E. Torruellas, G.I. Stegeman, Opt. Lett. 22, 19 (1997).

[25] D.A. Kleinman, A. Ashkin, G.D. Boyd, Phys. Rev. 145, 338 (1966).

[26] G.D. Boyd, D.A. Kleinman, J. Appl. Phys. 39, 3597 (1968).

[27] L. Torner, D. Mazilu, D. Mihalache, Phys. Rev. Lett. 77, 2455 (1996). 